${ }^{1}$ Department of Pediatrics, Diabetology, Endocrinology and Nephrology, Medical University of Lodz, Lodz, Poland

${ }^{2}$ Poznan University of Medical Sciences, Poznan, Poland

${ }^{3}$ Department of Pediatrics, Endocrinology, Diabetology, Metabolic Disorders, and Cardiology of Developmental Age,

Pomeranian Medical University, Szczecin, Poland

${ }^{4}$ Department of Metabolic Diseases, University Hospital, Jagiellonian University Medical College, Krakow, Poland

${ }^{5}$ Department of Hypertension and Diabetology, Medical University of Gdansk, Gdansk, Poland

${ }^{6}$ Department of Pediatrics, Diabetology and Endocrinology, Medical University of Gdansk, Poland

\title{
Experts opinion: implantable continuous glucose monitoring system - innovation in the management of diabetes
}

\section{ABSTRACT}

Continuous glucose monitoring (CGM) systems have revolutionized the treatment and monitoring of diabetes. These devices are recommended for diabetic patients treated with insulin, especially with recurring episodes of hypoglycemia or large circadian variation of glucose levels. CGM allows more effective adjustment of insulin doses to blood glucose trends, resulting in better metabolic control: more time spend in glucose target range, lower time spend in hypoglycemic range, lower glucose variability and improved quality of life of patients and their family members. Real time CGM provides patients not only with continuous information on glucose levels but also alerts for hypo- or hyperglycemic events.

Traditional transcutaneous CGM have some limitations, which can be resolved by the system with an implantable sensor. The Eversense CGM is the only long-term implantable rtCGM. The subcutaneous implantation procedure has proved to be simple and uncomplicated. This CGM system can be recommended in particular

\section{Address for correspondence:}

dr hab. n. med. Agnieszka Szadkowska

Klinika Pediatrii, Diabetologii, Endokrynologii i Nefrologii

Uniwersytet Medyczny w Łodzi

ul. Sporna 36/50, 91-738 Łódź

Phone: +48 426177791

Fax: +48, 4261777 98, mobile phone: +48 607145644

e-mail: agnieszka.szadkowska@umed.lodz.pl,

agnieszka.szadkowska@wp.pl

Clinical Diabetology 2019, 8, 6, 318-328

DOI: $10.5603 /$ DK.2019.0030

Received: 27.10.2019

Accepted: 09.12.2019 for patients who, due to their profession and sports discipline, cannot or do not want to use traditional transcutaneous sensors. Further groups are patients with skin complications associated with the use of traditional sensors, people who perceive frequent sensor replacement as a burden or would benefit from on-body vibration alerts. The studies showed that the patients were satisfied with Eversense, and the majority used it several times after the study ended. The main reason for resigning from the next implantation was cost of the device. (Clin Diabetol 2019; 8, 6: 318-328)

Key words: long-term implantable glucose monitoring, real-time continuous glucose monitoring system, clinical practice opinion

\section{Introduction}

The burden of diabetes is steadily increasing. Current estimations implicate that there are more than 450 million people with diabetes worldwide and almost half of them are undiagnosed. According to the International Diabetes Federation, it is expected that the prevalence of diabetes will rise to 693 million people by 2045 [1].

It is commonly known that chronic hyperglycemia can affect the structure and impair the function of many tissues in the body, especially the vascular system. Diabetic complications and comorbid conditions primarily determine the quality of life, and they are mainly responsible for the increased mortality of patients [2]. 
So far, the main marker which is used to evaluate the risk of long-term diabetes complications is glycated hemoglobin $\left(\mathrm{HbA}_{1 \mathrm{c}}\right) . \mathrm{HbA}_{1 \mathrm{c}}$ level is an important indicator of glycemic control. Regular $\mathrm{HbA}_{1 \mathrm{c}}$ level measurements are also helpful in the evaluation of diabetes treatment efficiency [3].

Nowadays, the overall goal of diabetes control in diabetic patients is $\mathrm{HbA}_{1 \mathrm{c}}$ level $\leq 7.0 \%$ [4]. Better long-term diabetes control resulting in a reduction in $\mathrm{HbA}_{1 \mathrm{c}}$ is associated with decreased risk of chronic complications and mortality [5]. However, it should be emphasized that $\mathrm{HbA}_{1 \mathrm{c}}$ level does not reflect glycemic variability, which emerged recently as another possible risk factor for vascular dysfunction in diabetes [6]. The limited control of glycemia drives also the higher risk of hypoglycemia. Fear of hypoglycemia results in maintaining elevated blood glucose levels and it is the important cause of insufficient metabolic control of the disease and reduces the possibility of treatment intensification [7]. Currently, hypoglycemia is considered to be the greatest obstacle to achieve metabolic control of diabetes. Moreover, severe hypoglycemia has been also considered to be one of the predictors of macrovascular events and also increased mortality in patients with diabetes.

The evaluation of glucose variability and the risk of hypoglycemia has a great impact on the management of diabetes. It allows assessment of the effectiveness of therapy and provides guidance in selecting the appropriate insulin dosage schedule.

Therefore, blood glucose monitoring and appropriate management of glycemia is important not only to prevent chronic complications by reducing hyperglycemia but also to avoid hypoglycemic episodes and to decrease glycemic variability $[8,9]$.

\section{Blood glucose monitoring as a part of the integral care of diabetic patients}

Current monitoring and retrospective evaluation of blood glucose levels are essential parts of adequate diabetes treatment [4]. BG monitoring allows patients to assess their response to the treatment, decrease the risk of hypoglycemia, and to determine whether they are achieving glycemic control. Detailed information about blood glucose levels can be helpful in the adjustments in therapy and lifestyle activities and simultaneously to prevent diabetes-related complications. This is typically achieved using conventional personal blood glucose meters to measure finger-prick capillary blood samples [10]. The recommended frequency of self-monitoring of blood glucose (SMBG) is mainly dependent on the type of diabetes, treatment regimen and susceptibility to hypoglycemia. Intensification of the treatment is associated with the need for more frequent blood glucose monitoring [4].

Regardless of the treatment used, all patients should check blood glucose levels in case of feeling unwell, a sudden illness or suspected hypoglycemia. They should monitor blood glucose before planned physical activity and before activities associated with particular dangers of hypoglycemia (e.g. driving).

However, testing six to eight or more times daily SMBG may burden patients and may result in noncompliance. Therefore, it is also recommended to ensure that patients are properly instructed and are given regular evaluation and follow-up. Proper SMBG requires patient education regarding glucose meter use, interpretation of readings, and further management steps [4].

Self-monitoring of blood glucose was the standard of care for patients with diabetes for a few decades. So far, it is a widely used method of current glucose monitoring in Poland. However, SMBG has notable limitations. It is insufficient to diagnose all acute episodes of hyper- and hypoglycemia and thereby to get a full, daily profile of glycemia, which can allow for rapid patient reaction or adjustment of diabetes treatment. A glucose meter measures glucose levels at a single moment in time, and therefore, it does not indicate the direction or rate of change of glucose levels. Using glucose meter data alone may result in inappropriate therapy decisions (such as administering correction insulin when blood glucose levels are falling). Accordingly, SMBG often fails to detect nocturnal and asymptomatic hypoglycemia [10, 11]. Moreover, obtaining glucose data via glucose meter is dependent upon the patient's decision to self-monitor. It requires a finger prick to obtain a blood sample, and it results in pain for the patient, which also affects patient compliance with glucose measurements.

\section{Continuous glucose monitoring}

The introduction of continuous glucose monitoring (CGM) systems in 1999 have slowly changed standards of medical care in diabetes. In recent years, blood glucose monitoring has been revolutionized by the development of different systems for continuous glucose monitoring (CGM).

Currently, there are two types of new technological devices available to measure glucose levels in the interstitial fluid through sensors inserted subcutaneously: real-time CGM and Flash Glucose Monitoring (FGM, intermittent scanning continuous glucose monitoring - is-CGM). Both systems provide information about current and previous glucose levels, as well as glucose trends and anticipated future glycemic status, but each 
technology has its unique features. FGM provides glucose information on demand. CGM measures glucose automatically and, as often as every five minutes, which generates 288 measurements per day. RtCGM provides for patients not only continuous current information on glucose levels in interstitial fluid over the whole day, but also alerts for hypo- or hyperglycemic events and rapid glucose trends [11].

\section{Comparison of CGM systems}

In Poland, there are a few real-time CGM systems available, e.g., Guardian ${ }^{\text {TM }}$ Connect (Medtronic), Dexcom G4, Dexcom G5 and the Eversense CGM. Patients who choose to use CGM have several options available to them, standalone devices or insulin pumps integrated with CGM (MiniMed ${ }^{\circledR}$ Real Time ${ }^{\text {TM }} 722$, MiniMed $^{\circledR}$ Veo $^{\mathrm{TM}}{ }^{\text {, MiniMed }}{ }^{\circledR}{ }^{640 \mathrm{G}^{\mathrm{TM}}}{ }^{\mathrm{M}}$ Medtronic) [12].

The Medtronic and Dexcom systems utilize transcutaneous sensors (transcutaneous real-time CGM - TC rtCGM). They consist of three components: a disposable sensor that is inserted into the subcutaneous tissue to measure glucose levels, a transmitter that attaches to the sensor, and a receiver (stand-alone device, insulin pump, smartphone, smartwatch) that displays and stores glucose information [12].

The Eversense CGM is the only, long-term implantable real-time CGM (LTI rtCGM). The system consists of an implantable, fluorescence-based, cylindrical glucose sensor, a removable external smart transmitter and a mobile medical application that displays glucose information and operates on a mobile device that allows users to review current and historical glucose data in real-time. The Eversense system is indicated for up to 180-days wear time in adults only [12].

FreeStyle Libre, the first flash glucose monitoring (FGM) system, was approved in Autumn 2014. FGM also measures glucose concentration in the interstitial fluid. However, it differs from other CGM technology in several ways. FGM is factory calibrated and does not require capillary blood glucose calibration. Sensor glucose levels are not continually displayed on a monitoring device but instead are displayed when the sensor is "flashed" with a reader device on demand. The FGM reader also displays a plot profile of the last 8 hours, derived from interpolating glucose concentrations recorded every 15 minutes. Therefore, when the patient with diabetes performs $\geq 3$ sensor scans per day at $\leq 8$-hour intervals, the FGM records 24-hour glucose profiles. The sensor can be worn continuously for up to 14 days, but it does not provide low or high glucose alarms in first-generation system. FGM system provides protection against hypoglycemia during the day, but it cannot detect nocturnal hypoglycemia when the user is sleeping or warn the physically active individual about pending hypoglycemia [13-15].

Also, all CGM systems can simultaneously transmit data to the cloud to share information in real-time. To date, insulin pumps integrated with CGM do not have such a function.

Jafri et al. compared available CGM by testing the performance of the Dexcom G5, Abbot Freestyle Libre Pro, and Senseonics Eversense during a 6-week, free life, outpatient bionic pancreas study involving 23 subjects with type 1 diabetes who wore all 3 devices concomitantly. The primary outcome was the mean absolute relative difference (MARD) vs. plasma glucose level measured with a glucometer. All 3 CGM systems produced higher average MARDs than during in-clinic studies. In the 3-way comparison Eversense achieved the lowest nominal MARD (14.8\%) followed by Dexcom G5 (16.3\%) and Libre Pro (18.0\%). The pointing accuracy of the Eversense was significantly better than two other CGM systems [16].

\section{Clinical use of continuous glucose monitoring}

CGM and FGM systems are becoming increasingly prevalent in clinical practice because using it can reduce patients' discomfort and provide vastly more detailed glucose variability data. These systems can supply insight into the duration, frequency of hypoand hyperglycemia and fluctuations in blood glucose levels. Therefore, they can be helpful to improve overall glycemic control by identifying episodes and preventing periods of hypoglycemia and hyperglycemia [15, 17].

Continuous glucose monitoring allows more effective adjustment of insulin doses to blood glucose trends, resulting in better metabolic control: more time spent in the glucose target range, a reduced number of hypoglycemia episodes (or lower time spend in hypoglycemic range), lower glucose variability and improved quality of life of patients and their caregivers [16].

According to the Diabetes Poland guidelines, the use of continuous glucose monitoring systems, including rtCGM and FGM is particularly indicated in patients with labile diabetes type 1 , in patients with frequent hypoglycemic episodes. RtCGM is particularly indicated in patients with hypoglycemia unawareness, frequent nocturnal hypoglycemia and children $<10$ years of age. In these patient groups, it is also recommended to use insulin pumps integrated with CGM, with a function of automatic temporary insulin suspension of the insulin infusion at low blood glucose values or at risk of hypoglycemia [4].

Initially, as recommended, patients using CGM prior to making therapeutic decisions should confirm the reading with a conventional meter. Currently, pa- 
Table 1. Decision algorithm for glucose monitoring according to Clinical Practice Recommendations on the Routine Use of Eversense [15]

\begin{tabular}{|c|c|c|c|c|}
\hline & SMBG & isCGM/FGM & TC rtCGM & LTI rtCGM \\
\hline Intensified insulin therapy & + & + & + & + \\
\hline \multicolumn{5}{|l|}{ High glycemic variability } \\
\hline Committed to improved self-management & & + & + & + \\
\hline \multicolumn{5}{|c|}{ Treatment goals not achieved despite intensive treatment and training } \\
\hline Frequent hypoglycemia & & & + & + \\
\hline \multicolumn{5}{|l|}{ Unawareness of hypoglycemia } \\
\hline \multicolumn{5}{|l|}{ Fear of pain or needle phobia } \\
\hline \multicolumn{5}{|c|}{ Physically handicapped (visual, hearing, dexterity impairment) } \\
\hline History of skin problems & & & & + \\
\hline \multicolumn{5}{|l|}{ Need for vibration alerts } \\
\hline Need for transient removal of external devices & & & & \\
\hline
\end{tabular}

isCGM — intermittent scanning continuous glucose monitoring; LTI rtCGM — long-term implantable real-time CGM; SMBG — self-monitoring of blood glucose; TC rtCGM - transcutaneous real-time CGM

tients can make therapeutic decisions based on the Dexcom G5, the Dexcom G6, FGM. In June 2019, the FDA approved a nonadjunctive indication for the 90 days system Eversense CGM. The FDA reviewed device performance data and proposed changes to the Eversense App (that support non-adjunctive use) and determined that Eversense is safe for making treatment decisions (such as dosing insulin and consuming carbohydrates) based on CGM glucose readings and trend arrows.

Szypowska et al. conducted a meta-analysis of seven RCTs to explore the potential beneficial effects of the use of real-time CGM in patients with type 1 diabetes mellitus. This analysis showed that the use of real-time CGM for over $60-70 \%$ of time provides a superior benefit over self-monitoring of blood glucose concerning $\mathrm{HbA}_{1 \mathrm{c}}$ reduction. The improvement in $\mathrm{HbA}_{1 \mathrm{c}}$ in patients using real-time CGM was achieved without an increase in severe hypoglycemia. The reduction in $\mathrm{HbA}_{1 \mathrm{c}}$ was noted not only in patients with poorly controlled type 1 diabetes but also in well-controlled subjects. The superiority of real-time CGM over SMBG in lowering $\mathrm{HbA}_{1 c}$ was also confirmed in pump users [18].

In general, the choice of an optimal glucose monitoring device should be dependent on the patient's clinical indications or lifestyle restrictions [13, 19]. Patients treated with intensive insulin therapy have a wide choice of glucose monitoring devices according to their personal preferences. The proposition of the decision algorithm for the use of specific devices for glucose monitoring was presented in Table 1 [20].

\section{Education process of patients and physicians about optimal using of CGM}

CGM systems provide real-time data on interstitial glucose level, direction and rate of change in blood glucose levels. However, users of CGM may not be able to make optimal usage of this additional information without proper education. The appropriate interpretation of measured parameters to make correct therapeutic decisions is very important. Therefore, using CGM has to be supported with education of patients. Use of CGM/FGM requires structured education regarding appropriate expectations towards this system and proper interpretation of current readings including glucose trends [4].

The training for rtCGM should also include the principles of system functioning, its calibration and placing a sensor. Multiple alarms can be set to alert users if blood glucose increases or drops beyond defined target ranges. The most successful education programs emphasize training on self-management, including specific rules to adjust the insulin dose based on glucose data. Patients who understand how to interpret trend arrows have the best outcomes. At the time, when the trend arrow indicates a falling glucose level, then carbohydrates should be consumed, or dose of the prandial insulin should be decreased, and when the trend arrow indicate a rising glucose level, then the dose of insulin should be increased or physical activity should be started. Optimal using of CGM allows the patients to make lifestyle adjustments and therapeutic changes to improve their glycemic control [11].

The optimal training includes three parts: the principles of sensor technology, the operational aspects of the device, and the interpretation of the provided data. This training should commence 1 week before starting using the system.

Also, some physicians lack the appropriate level of knowledge to use CGM as part of their practices. There is need for considerable education regarding inter- 
pretation of CGM results for the physicians and other healthcare workers. Educational programs focused on these knowledge and coverage gaps will enable professionals to provide improved care of diabetic patients [11]. According to the Recommendations from the International Consensus on Time in Range, both medical staff and patients should know clinical targets for CGM data interpretation [21].

\section{Senseonics Eversense CGM System Description of the system and insertion procedure}

Eversense is a novel implantable subcutaneous CGM system produced by Senseonics Inc. It was designed to address several of the currently available CGM systems limitations. Eversense CGM sensor is the first one approved for long-term use and it can monitor blood glucose levels every five minutes for up to 90-180 days, and thus it allows to reduce the inconvenience and discomfort of frequent sensor insertion procedures. Eversense CGM system differs from other systems currently on the market in that the sensor is implanted subcutaneously by a doctor; the removable smart transmitter is placed on the skin over the sensor and can be taken on and off as desired by the patient [22].

CGM systems based on electrochemical- and enzymatic- methods are often a subject of interference with substances such as ascorbic acid, paracetamol, dopamine or maltose. Eversense is a fluorescence-based, therefore, these substances do not affect the sensor readings. It has also a silicone ring that contains an anti-inflammatory steroid drug (dexamethasone acetate). The system provides continuous glucose measurements over a $40-400 \mathrm{mg} / \mathrm{dL}$ range. The sensor requires twice-daily calibration. The Eversense smart transmitter is worn over the sensor and wirelessly powers it to initiate the glucose measurement and the transfer of data to the Mobile Medical Application (MMA). The transmitter can be removed at any time without the need for sensor replacement, allowing greater convenience and lifestyle flexibility. In addition, hypoglycemic and hyperglycemic alerts and notifications are provided on a mobile device. Additionally, the patient experiences on-body vibratory alerts from the transmitter even when the mobile device is not nearby. Thus, Eversense CGM system gives the desired flexibility and freedom to test blood glucose anywhere at any time and share and analyze their personalized data securely via a smart app. It is also water-resistant submerged in 1 meter for up to 30 minutes and it functions perfectly while the users take a shower or swim. The Eversense Mobile App runs on a compatible mobile device to receive and display the sensor glucose data from the Eversense Smart Transmitter. It eliminates the need to carry a separate receiver device. However, it is also important to check phone compatibility for CGM mobile applications before insertion. It is also able to set up a temporary glucose profile with custom high and low target and alert levels [22].

The Eversense CGM System Kit comes in three packages: the sensor pack, the insertion tools pack, and the smart transmitter pack. The sensor is shipped sterile inside a protective holder for safe handling purposes. The Eversense insertion tools pack contains the incision template, blunt dissector, insertion tool and adhesive patches. The incision template is used to guide and mark the incision area on the skin surface by aligning the marking template to the marked outer edges of the smart transmitter when placed in a comfortable position. The suggested insertion location is approximately at the midway point between the acromion process and the lateral epicondyle. The insertion should avoid areas with loose skin (such as back of arm), scar tissue, tattoo or nevus. During the procedure, the patient should be positioned in a reclined position, preferably on their side, with their elbow flexed up to 90 degrees and their palm resting on their chest or abdomen. The insertion area should be cleaned and disinfected before the application. Local anesthetic should be injected approximately $5 \mathrm{~mm}$ along the planned incision and approximately $30 \mathrm{~mm}$ perpendicular to the planned incision which is the planned canal of the blunt dissector tool [22].

The attached blunt dissector (Fig. 1A) is used to create the subcutaneous pocket for insertion of the sensor. This tool has two depth guards to help prevent the pocket from being made too deep in the skin. The depth guards have guide marks to assist in determining the length of the subcutaneous pocket for placing the sensor. The insertion should be made approximately $5 \mathrm{~mm}$ at the insertion location in such way that it will be able to create an appropriately sized subcutaneous pocket approximately $3-5 \mathrm{~mm}$ below the skin surface. The blunt dissector should be moved toward the shoulder while maintaining the metallic and plastic parts of the tool in close contact with the skin to ensure the smallest possible angle of the pocket with respect to the skin. The pocket should not be created more than 3-5 mm below the skin. If the sensor is placed too deep, it may be difficult to communicate with the smart transmitter or to be removed, therefore recent changes made to the blunt dissector should prevent placement mistakes [22].

To insert the sensor inside the subcutaneous pocket, the insertion tool has to be used. It has two guide marks on the cannula to assist in proper placement. 


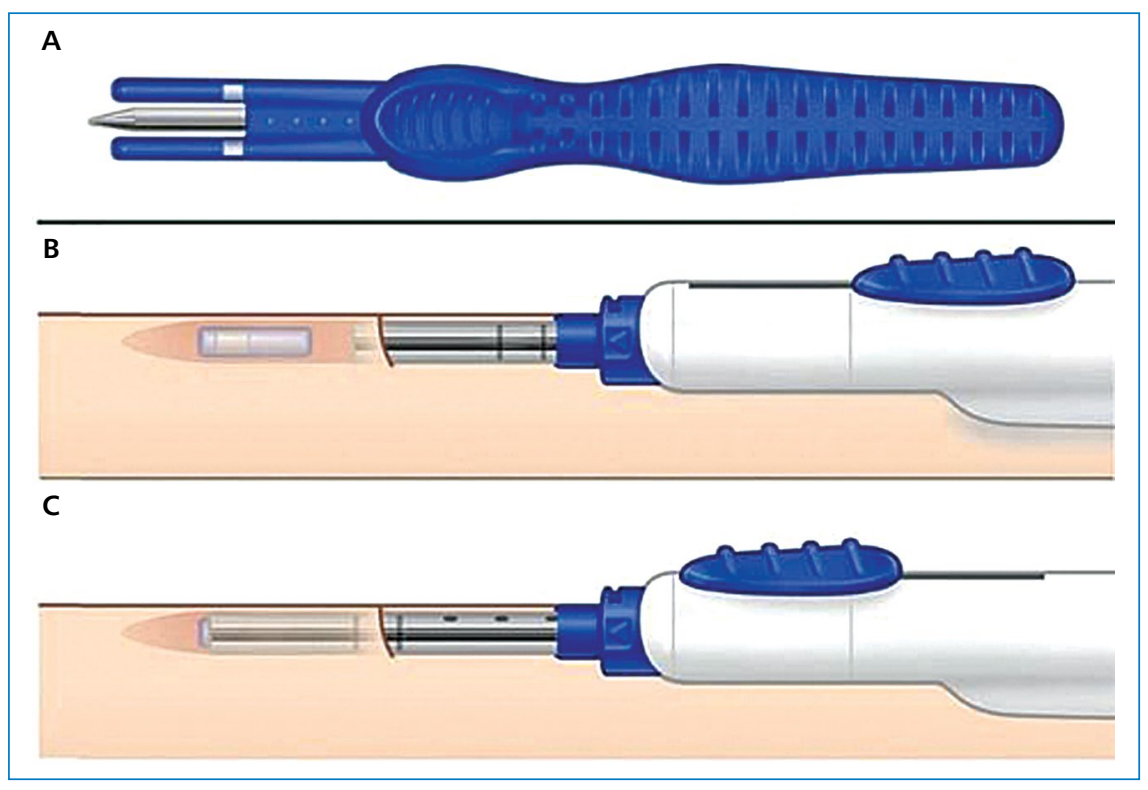

Figure 1. Insertion tools provided with the Eversense system

The tip of the insertion tool should be placed into the incision opening. Firstly, the insertion tool has to be unlocked by a pushing down on the back of the thumb slide (Fig. 1B). The sensor is deployed into the pocket by a retracting of the thumb slide (Fig. 1C). Before removing the insertion tool from the incision, the insertion area should be lightly palpated to confirm that the sensor is in place. The incision should be closed and dressed in the appropriate manner using adhesive skin closure or suture and dressing, making sure the two sides of the incision are closed together. Adhesive patches, included with the Eversense insertion tools pack, have an adhesive side that attaches to the back of the smart transmitter and a silicone adhesive side that attaches to the skin. It is important to inform the patient that adhesive patches should be replaced daily [22].

Even though, at first sight, the required minimally invasive procedure exceeds the area of experience for many diabetologists, the technique is easy to learn when performed with proper training, oversight, and attention to detail. Therefore, any physician interested in undertaking the procedure should be accompanied and consequently certified by the company's clinical training manager during the first several insertions and removals to ensure the required training to comply with the highest quality standards [20]. Recently in Poland, there are 32 doctors who have sufficient experience ( 3 training insertions of a sensor) to carry out of implementation procedure. Furthermore, 27 doctors carried out 3 or more insertions and removal of the Eversense system and thus obtained a full certificate.
Above procedure can be carried out in one of 30 clinics or facilities, especially in large cities.

\section{Clinical experience}

Long-term accuracy and clinical utility of Eversense system have been validated in prospective clinical trials (Table 2). In the multinational, multicenter European PRECISE trial, the safety and accuracy of the Eversense CGM system was investigated. It also assessed sensor lifetime, system wearout time, participant reported outcome measures, and parameters of glycemic control. The Eversense system was studied in 71 participants aged 18 years and older with type 1 and type 2 diabetes during 180 days. CGM accuracy was assessed during eight in-clinic visits with the mean absolute relative difference (MARD) for venous reference glucose values $>4.2 \mathrm{mmol} / \mathrm{L}$. The MARD value was $11.1 \%$ and $81 \%$ of hypoglycemic events were detected by the CGM system within $30 \mathrm{~min}$. $\mathrm{HbA}_{1 \mathrm{c}}$ improved in the study group from $7.54 \%$ (59 mmol $/ \mathrm{mol}$ ) at baseline to $7.19 \%$ (55 mmol/ $/ \mathrm{mol}$ ) at the end of study. No device-related serious adverse events occurred during the study. The results from this study indicated that the use of a long-term Eversense CGM sensor is both effective and safe and provides specific usability benefits [23].

Barnard et al. determined acceptability and impact of an Eversense CGM sensor (as part of the PRECISE trial). Fifty-one participants took part in the study. Quantitative psychosocial assessments were administered at 90 days to participants to explore patient-reported outcomes associated with an implanted sensor. 
Table 2. Parameters characterizing the accuracy and safety of the Eversense CGM (based on results of clinical trials)

\begin{tabular}{|c|c|c|c|c|c|}
\hline Study & $\begin{array}{c}\mathrm{HbA}_{1 \mathrm{c}} \text { reduction } \\
\text { Overall } \mathrm{HbA}_{1 \mathrm{c}} \\
\text { level }\end{array}$ & $\begin{array}{c}\text { Accuracy (MARD) } \\
\text { Overall (\%) } \\
40-400 \mathrm{mg} / \mathrm{dl}\end{array}$ & $\begin{array}{c}\text { Adherence } \\
\text { Hours per day }\end{array}$ & $\begin{array}{l}\text { Time in target } \\
\text { Overall (\%) } \\
70-180 \mathrm{mg} / \mathrm{dl}\end{array}$ & $\begin{array}{c}\text { Hypoglycemic } \\
\text { detection } \\
\text { Overall (\%) } \\
70 \mathrm{mg} / \mathrm{dl}\end{array}$ \\
\hline PRECISE 1 & $0.4 \%$ & $11.6 \%$ & 23.5 & $59.9 \%$ & $81 \%$ \\
\hline PRECISE 2 & $0.5 \%$ & $8.5 \%$ & 23.4 & $57.6 \%$ & $93 \%$ \\
\hline PRECISION & $0.3 \%$ & $9.6 \%$ & 23.4 & $59.0 \%$ & $95 \%$ \\
\hline
\end{tabular}

Key issues included impact of device on perceptions of diabetes self-management and diabetes control, usability, safety, social relationships, and fear of hypoglycemia. The system was rated highly on ease of use, convenience, and comfort. CGM Impact Scale results showed that $86 \%$ of participants reported feeling better about their diabetes control. Furthermore, $73 \%$ felt safer while sleeping and $78 \%$ more confident about avoiding serious hypoglycemia. It was concluded that the Eversense CGM sensor was acceptable to participants and use of the system was associated with minimized burden of diabetes [24].

In 2018, there were also results of PRECISE II trial published. It was a nonrandomized, blinded, prospective, single-arm, multicenter study that evaluated the accuracy and safety of the Eversense CGM system among adult participants with T1D and T2D. Ninety participants were enrolled and each received the CGM system. The primary endpoint was the MARD between paired Eversense and reference measurements through 90 days post insertion. The overall MARD value against reference glucose values was $8.8 \%$, which was significantly lower than the prespecified $20 \%$ performance goal for accuracy. The system had a favorable safety profile for its intended use. Clinicians with limited to no surgical experience were able to insert and remove the sensor without difficulty after appropriate training. The results of PRECISE II trial demonstrated that the use of Eversense sensor for a long-term is accurate and safe [25].

Aronson et al. conducted a prospective, singlecentre, single-arm, 180-day study to evaluate the effectiveness and safety of the implantable CGM system in adolescent and adult subjects with T1D. Accuracy measures included mean absolute relative difference (MARD), 15/15\% agreement between CGM glucose and blood glucose measured by SMBG and surveillance error grid analysis. Overall MARD was $9.4 \%$. CGM system agreement at 15/15\% through 60, 120 and 180 days was $82.9 \%, 83.6 \%$ and $83.4 \%$, respectively. Surveillance error grid analysis showed $98.4 \%$ of paired values in clinically acceptable error zones. No insertion/removal or device-related serious adverse events were reported. Results of above study confirmed that the Eversense XL CGM system is safe and accurate through 180 days in a primarily adolescent population of subjects with T1D [26].

\section{Clinical experience in Poland}

We have also collected information on experience with Eversense CGM in various clinical centers in Poland.

First implantation of the Everense sensor was made on 22 October 2017 in Raszeja Hospital in Poznan (Department of Internal Medicine and Diabetology Poznań University of Medical Sciences). The implantation/reimplantation procedure is performed in an outpatient clinic. An earlier qualifying visit is indicated during which the patient's expectations and possible contraindications to the procedure are discussed. The procedure along with the preparation of the patient takes about 20-30 minutes. The implanting time usually does not exceed 15 minutes. The removal of the sensor is less predictable in time. It can last very short, but it can also be extended up to 30 minutes, especially when it is necessary to locate the sensor using an ultrasound scanner. Performing over 80 procedures of implantation and reimplantation, no complications were found. In no case did the wound require sewing. There was no infection, hematoma, non-healing wound, sensory disturbances, skin changes, allergic reaction. During one removal operation, ultrasound was used to find the sensor. Some patients were examined by physician 2 days after surgery to evaluate and change the dressing. In one case it was required to replace the steri-strips. Most of the patients stayed in contact with their doctors via telephone. Steri-strip were removed by patients themselves on the $6-7^{\text {th }}$ day after the procedure. Regardless of whether the patients used insulin pen or a personal insulin pump, everyone was satisfied with Eversense. The majority, despite emphasizing the discomfort of the implantation procedure, had it carried out several times. Patients who resigned from the next implantation usually did so for financial reasons.

In the Central Clinical Hospital of the Medical University of Lodz the Eversense sensor implementation procedure was introduced in January 2018. So far, over 80 procedures of implantation, removal and 
reimplantation of sensors have been performed. This CGM system was offered mainly to patients who, due to their profession and sports discipline, could not or did not want to use traditional transcutaneous sensors. Further groups were patients with skin reactions associated with the use of traditional sensors or patients who perceive frequent sensor replacement as a burden or benefited from on-body vibration alerts. 17 patients are now using the third sensor. The main reason for not continuing this CGM system is the price of the device. In the opinion of doctors from this centre, the sensor implementation was very simple and not a time consuming procedure. Removal of the sensor was usually an easy and short procedure. Problems while removing the sensor occurred only in two patients. Both patients required more lignocaine for anaesthesia and both needed ultrasonography to locate the sensor.

The next clinical experiences came from Clinical Diabetology Center in Krakow. The center has been implanting the sensors since August 2018 in patients with diabetes coming to the Clinic or directed by doctors from the Malopolska province. So far, 25 successful implantations and 13 sensor exchanges have been carried out. There were no complications both for implantation and replacement. The subcutaneous session implantation procedure with the provided tools proved to be simple and uncomplicated. Also, there were no difficulties in attaching the sensor, it is easy to feel the sensor by palpation. Re-establishment of the sensor is carried out on the other arm. Patients were eager to use the CGM system. Eversense CGM system was mostly indicated for patients with a fear of hypoglycemia, glycemic instability and aversion to frequent glycemic measurements in self-control, poor tolerance of traditional transcutaneous sensors or for patients practicing competitive sports.

In all centers, no permanent complications were found during the implementation or removal of the sensors. In two patients a transient slight lipodystrophy was observed after sensor removal. In one case, the wound was sewn together at the request of a very sport active patient.

In the opinion of doctors from all centres, the use of Eversense contributed to the improvement of the effects of therapy, expressed as the time spent in the target blood glucose values. They emphasize the comfort and usefulness for the patients practicing sports.

\section{Patients experience in Poland}

Roche Diabetes Care with the cooperation of Biostat, conducted a study to investigate the patients' opinion about Eversense CGM system [27]. The study was aimed at gathering information about the Ever- sense system and the impact of CGM on the life of a patient with diabetes. Advantages and disadvantages of the system and its individual components have also been evaluated. There were 86 enrolled patients who used the Eversense system for three months and then answered questions in the questionnaire. The study included almost as many men as women (48.8\% vs. $51.2 \%)$. Among the respondents, the largest group were patients aged $30-39$ (27.9\%). About $22.1 \%$ of the respondents were between 18 and 29 years old. The smallest group of patients were people aged 50-59 (5.8\%). About half of the patients $(51.2 \%)$ had prior experience with CGM system.

Patients were also asked to rate the system components. In one of the questions, patients had to evaluate the sensor's activity time. Every third respondent (33.7\%) rated the sensor activity very well, and 39.5\% assessed it well. According to $16.3 \%$ of respondents, the sensor activity time was medium, and respectively $7 \%$ and $2.3 \%$ of the respondents considered this element of the system to be rather weak and weak. However, it should be emphasized that patients used a previous version of sensor which was implanted for 3 months. Over three fourth of the respondents rated the alarms positively: $37.2 \%$ very well and $41.9 \%$ good. About $11 \%$ of patients considered it as medium, $4.6 \%$ as rather weak, and 3.5\% as weak.

One of the reasons for traditional CGMs limited use include a perceived burden of frequent insertions, fear of pain or discomfort during an implantation procedure. Therefore, patients were asked to evaluate a method for inserting sensor. Over half of the respondents considered the insertion of the sensor as very good (54.6\%), moreover, according to $30.2 \%$ people, this method was good. About $8.1 \%$ of patients assessed the insertion of the sensor on medium rate, $5.8 \%$ as rather poorly and only $1.2 \%$ poorly.

Education is the core to fully understanding and integrating a CGM into daily practice and patients' lifestyles. Training on CGM is important to ensure that patients know how to use the device and set the appropriate expectations. The educational training in the use of system operation received particularly positive marks. As many as $83.7 \%$ of people evaluated the training very well, and $15.1 \%$ of people - well. Only one person (1.2\%) considered the training to be poor.

Patients were also asked to answer the question: "What benefits do you see in using the Eversense system for therapy?". More than 34\% of respondents indicated the possibility of continuous control and access to blood glucose level measurements. About $17.9 \%$ of patients indicated benefits related to warning alarms. Other significant benefits of the system in the respond- 
ents' opinion were: displaying glucose trends (10.1\%), limited number of SMBG measurements (7.7\%), userfriendly application or the possibility of glycemic control during physical activities or sleep (5.4\%).

However, most importantly, $87.2 \%$ of respondents confirmed that the use of the Eversense system had a positive impact on their daily life with diabetes. Respondents indicated that the use of the Eversense CGM system allows for easy glycemic monitoring, better maintaining of target glucose level and improvement of the quality of life (each of these answers were indicated by $15.4 \%$ of patients). The reduction in the number of finger pricks was indicated by $12.5 \%$. About $5 \%$ of patients appreciated the usefulness of Eversense system in the prevention of hypo- and hyperglycemia and assistance in the selection of meals. Almost $4 \%$ of people indicated safety, psychological comfort, more precise adjustment of insulin doses and the ability to monitor blood glucose even at night.

Despite good ratings of the Eversense CGM system, only $39.5 \%$ of patients declared that they intend to continue using the Eversense system. Patients were asked why they do not intend to use Eversense system. The vast majority of patients $(65.1 \%)$ indicated a too high price and lack of reimbursement.

\section{Global reimbursement of CGM}

Clinical studies demonstrated that the use of CGM could reduce hyperglycemia and hypoglycemia episodes by providing the patients information about blood glucose levels as well as the rate and direction of glycemia changes. Randomized controlled trials have shown that these electronic devices can be also helpful in lowering the $\mathrm{HbA}_{1 \mathrm{c}}$ level without increasing the risk of hypoglycemia in patients with T1DM. Despite the fact that the evidence base for CGM clinical efficacy has grown, coverage by global reimbursement authorities is still limited. It is associated with a lack of independent, robust, randomized clinical trials demonstrating both improved outcomes for hyperglycemia and hypoglycemia in specific patient populations. Moreover, there is only limited data on cost-effectiveness of CGM systems published. Reimbursement by payers is critical for the uptake and use of new diabetes technologies which can decrease the risk of acute and chronic complications of diabetes. Assuming that the daily costs for CGM usage are of approximately $\$ 5-10$ per day, this account for $\$ 3,000$ per year per patient. In some European countries the cost is around 4,000€ per year $[28,29]$.

However, recently CGM is reimbursed in the United States. In the US, national health insurance program (Medicare) covers CGM and related supplies instead of blood glucose meters for making diabetes treatment decisions. Coverage criteria include intensively insulin treated patients who perform four or more SMBG/day, are taking multiple insulin injections or are using an insulin pump, and will require frequent adjustment of insulin dose based on the reading from CGM [29].

CGM sensors are also reimbursed in many European countries. In England, National Institute for Health and Care Excellence (NICE) published clinical guidelines covering the management of type 1 diabetes in adults and children. It was recommended that CGM should be offered to people with challenging or severe hypoglycemia, to those with impaired awareness or fear of hypoglycemia, and to those for whom self-monitoring of blood glucose has failed to achieve optimum results. Recently, in Germany, real time CGM systems are reimbursed for T1DM and T2DM treated with insulin. In a few European countries (e.g., Slovenia, Spain), reimbursement of CGM is limited to pediatric population [29].

\section{CGM reimbursement in Poland}

Reimbursement of CGM in Poland is still limited. The National Health Fund covers the rtCGM for children and adults under 26 years old with T1DM, treated with insulin pump. Coverage criteria only include patients with hypoglycemia unawareness (lack of prodromal symptoms of hypoglycemia after alcohol consumption was excluded) [30].

Unfortunately, current regulations prevent patients from accessing the Eversense CGM system in this patient group. This provision stipulates that the limit for sensors is PLN 600 per month, up to 4 pieces per month and a patient surcharge of $30 \%$ of the limit value. In the current wording, both the description of the medical device, the limit of financing from public funds and the period of use have been formulated without the fact that other CGM systems with a period of use longer than a week are already available on the Polish market. This provision prevents the reimbursement concerning Eversense CGM from being carried out and settled by the National Health Fund.

FGM is reimbursed only for children from 4 to 18 years old with type 1 diabetes mellitus with very well monitored blood glucose, i.e. at least 8 blood glucose measurements per day [31].

It should be emphasized that huge proportions of the adult T1DM population are identified with persistent poor glycemic control (glucose level fluctuations, hypoglycemia episodes). Limited reimbursement influences the limited access of patients with diabetes to these devices and could worsen their prognosis. 


\section{Conclusions}

Continuous glucose monitoring systems have revolutionized the treatment and monitoring of diabetes. These devices are especially recommended for diabetic patients treated with insulin. According to Diabetes Poland guidelines, use of CGM is indicated in patients with recurrent episodes of severe hypoglycemia or large circadian variation of blood glucose levels. CGM systems provide patients detailed information about the level of glucose and thus can reduce the risk of hypoglycemia and improve patient quality of life.

Eversense CGM is the only one system which is approved for long-term use (up to 180 days). The subcutaneous session implantation procedure proved to be simple and uncomplicated. This CGM system can be recommended in particular to patients who, due to their profession and sports discipline, cannot or do not want to use traditional transcutaneous sensors. Further groups are the patients with skin reactions associated with the use of traditional sensors or people who perceive frequent sensor replacement as a burden or benefited from on-body vibration alerts.

\section{Authors' contributions}

The authors are members of the expert panel of Roche Diabetes Care. The expert group was established by Roche Diabetes Care to provide opinion about the Eversense CGM system as a part of the meetings of advisory committee, which took place in Warsaw on October 27, 2017; December 12, 2017 and on March 09, 2018. This article describes the clinical practice of the authors, which was independent of any influence by the manufacturer and Roche Diabetes Care. The writing of the article was supported by Roche Diabetes Care, and certain information was provided by the manufacturer, Senseonics, Inc., while the authors were primarily responsible for the substance of the article and maintained full control over the final content. All authors and Roche reviewed and approved the article.

\section{Conflict of Interest}

A.Sz., B.W., A.G., M.M.: Advisory Board for: Abbott, Medtronic, Roche; founded Research for: Roche; sponsored lectures/seminars for: Medtronic, Roche, Abbott, D.Z.Z.: Advisory Board for: Abbott, Medtronic, Roche, sponsored lectures/seminars for: Medtronic, Roche, Abbott. K.C.: Advisory Board for: Medtronic, Roche; founded Research for: Medtronic, Roche; sponsored lectures/seminars for: Medtronic, Roche. MW declared no conflict of interest.

\section{REFERENCES}

1. Cho NH, Shaw JE, Karuranga S, et al. IDF Diabetes Atlas: Global estimates of diabetes prevalence for 2017 and projections for 2045. Diabetes Res Clin Pract. 2018; 138: 271-281, doi: 10.1016/j. diabres.2018.02.023, indexed in Pubmed: 29496507.

2. Papatheodorou K, Banach M, Bekiari E, et al. Complications of Diabetes 2017. J Diabetes Res. 2018; 2018: 3086167, doi: 10.1155/2018/3086167, indexed in Pubmed: 29713648.

3. Sherwani SI, Khan HA, Ekhzaimy A, et al. Significance of HbA1c Test in Diagnosis and Prognosis of Diabetic Patients. Biomark Insights. 2016; 11: 95-104, doi: 10.4137/BMI.S38440, indexed in Pubmed: 27398023.

4. Araszkiewicz A, Bandurska-Stankiewicz E, Budzyński A, et al. 2019 Guidelines on the management of diabetic patients. A position of Diabetes Poland. Clinical Diabetology. 2019; 8(1): 1-95, doi: 10.5603/dk.2019.0001.

5. Diabetes Control and Complications Trial (DCCT)/Epidemiology of Diabetes Interventions and Complications (EDIC) Study Research Group. Mortality in Type 1 Diabetes in the DCCT/EDIC Versus the General Population. Diabetes Care. 2016; 39(8): 1378-1383, doi: 10.2337/dc15-2399, indexed in Pubmed: 27411699.

6. Škrha J, Šoupal J, Škrha J, et al. Glucose variability, HbA1c and microvascular complications. Rev Endocr Metab Disord. 2016; 17(1): 103-110, doi: 10.1089/dia.2013.0205.

7. Shafiee G, Mohajeri-Tehrani M, Pajouhi M, et al. The importance of hypoglycemia in diabetic patients. J Diabetes Metab Disord. 2012; 11(1): 17, doi: 10.1186/2251-6581-11-17, indexed in Pubmed: 23497433.

8. Kalra S, Mukherjee JJ, Venkataraman S, et al. Hypoglycemia: The neglected complication. Indian J Endocrinol Metab. 2013; 17(5): 819-834, doi: 10.4103/2230-8210.117219, indexed in Pubmed: 24083163.

9. Yun JS, Park YM, Han K, et al. Severe hypoglycemia and cardiovascular or all-cause mortality in patients with type 2 diabetes. Diabetes Metab J. 2016; 40(3): 202-210, doi: 10.4093/ dmj.2016.40.3.202, indexed in Pubmed: 27098504.

10. Czupryniak L, Barkai L, Bolgarska S, et al. Self-monitoring of blood glucose in diabetes: from evidence to clinical reality in Central and Eastern Europe - recommendations from the international Central-Eastern European expert group. Diabetes Technol Ther. 2014; 16(7): 460-475, doi: 10.1089/dia.2013.0302, indexed in Pubmed: 24716890.

11. Klonoff DC, Ahn D, Drincic A. Continuous glucose monitoring: A review of the technology and clinical use. Diabetes Res Clin Pract. 2017; 133: 178-192, doi: 10.1016/j.diabres.2017.08.005, indexed in Pubmed: 28965029.

12. Klimek M, Tulwin T. Continuous glucose monitoring: review of promising technologies. MATEC Web of Conferences. 2019; 252: 02012, doi: 10.1051/matecconf/201925202012.

13. Edelman SV, Argento NB, Pettus J, et al. Clinical implications of real-time and intermittently scanned continuous glucose monitoring. Diabetes Care. 2018; 41(11): 2265-2274, doi: 10.2337/ dc18-1150, indexed in Pubmed: 30348844.

14. Heinemann L, Freckmann G. CGM versus FGM; or, continuous glucose monitoring is not flash glucose monitoring. J Diabetes Sci Technol. 2015; 9(5): 947-950, doi: 10.1177/1932296815603528, indexed in Pubmed: 26330484.

15. Mancini G, Berioli MG, Santi E, et al. Flash glucose monitoring: a review of the literature with a special focus on type 1 diabetes. Nutrients. 2018; 10(8), doi: 10.3390/nu10080992, indexed in Pubmed: 30060632.

16. Jafri R, Balliro C, El-Khatib F, et al. et al.. A Three-Way Accuracy Comparison of the Dexcom G5, Abbott Freestyle Libre Pro, and SenseonicsEversense CGM Devices in an Outpatient Study of Subjects with Type 1 Diabetes. Diabetes Jul. 2018; 67(Suppl 1): 14-OR.

17. Petrie JR, Peters AL, Bergenstal RM, et al. Improving the clinical value and utility of CGM systems: issues and recommendations: 
A joint statement of the European Association for the Study of Diabetes and the American Diabetes Association Diabetes Technology Working Group. Diabetologia. 2017; 60(12): 2319-2328, doi: 10.1007/s00125-017-4463-4, indexed in Pubmed: 29067486.

18. Szypowska A, Ramotowska A, Dzygalo K, et al. Beneficial effect of real-time continuous glucose monitoring system on glycemic control in type 1 diabetic patients: systematic review and metaanalysis of randomized trials. Eur J Endocrinol. 2012; 166(4): 567-574, doi: 10.1530/EJE-11-0642, indexed in Pubmed: 22096111.

19. Rodbard D. Continuous glucose monitoring: a review of successes, challenges, and opportunities. Diabetes Technol Ther. 2016; 18 Suppl 2: S3-SS13, doi: 10.1089/dia.2015.0417, indexed in Pubmed: 26784127.

20. Deiss D, Szadkowska A, Gordon D, et al. Clinical Practice Recommendations on the Routine Use of Eversense, the First Long-Term Implantable Continuous Glucose Monitoring System. Diabetes Technol Ther. 2019; 21(5): 254-264, doi: 10.1089/dia.2018.0397, indexed in Pubmed: 31021180.

21. Battelino T, Danne T, Bergenstal RM, et al. Clinical targets for continuous glucose monitoring data interpretation: recommendations from the international consensus on time in range. Diabetes Care. 2019; 42(8): 1593-1603, doi: 10.2337/dci19-0028, indexed in Pubmed: 31177185.

22. https://www.eversensediabetes.com/.

23. Kropff J, Choudhary P, Neupane S, et al. Accuracy and Longevity of an Implantable Continuous Glucose Sensor in the PRECISE Study: A 180-Day, Prospective, Multicenter, Pivotal Trial. Diabetes
Care. 2017; 40(1): 63-68, doi: 10.2337/dc16-1525, indexed in Pubmed: 27815290.

24. Barnard KD, Kropff J, Choudhary P, et al. Acceptability of implantable continuous glucose monitoring sensor. J Diabetes Sci Technol. 2018; 12(3): 634-638, doi: 10.1177/1932296817735123, indexed in Pubmed: 28990436.

25. Christiansen MP, Klaff $\sqcup$, Brazg R, et al. A Prospective Multicenter Evaluation of the Accuracy of a Novel Implanted Continuous Glucose Sensor: PRECISE II. Diabetes Technol Ther. 2018; 20(3): 197-206, doi: 10.1089/dia.2017.0142, indexed in Pubmed: 29381090.

26. Aronson R, Abitbol A, Tweden KS. First assessment of the performance of an implantable continuous glucose monitoring system through 180 days in a primarily adolescent population with type 1 diabetes. Diabetes Obes Metab. 2019; 21(7): 1689-1694, doi: 10.1111/dom.13726, indexed in Pubmed: 30938036.

27. Study of patients' opinion on Eversense system which allows continuous glucose monitoring (CGM). Biostat, final report, Warsaw, 08.2018 (data not published).

28. Heinemann L, DeVries JH. Reimbursement for continuous glucose monitoring. Diabetes Technol Ther. 2016; 18 Suppl 2: S248-S252, doi: 10.1089/dia.2015.0296, indexed in Pubmed: 26784130.

29. Graham C. Continuous glucose monitoring and global reimbursement: an update. Diabetes Technol Ther. 2017; 19(S3): S60-S66, doi: 10.1089/dia.2017.0096, indexed in Pubmed: 28585871.

30. Regulation of the Minister of Health from January 18, 2018. Dz.U. 2018 poz. 281.

31. Regulation of the Minister of Health from September 26, 2019. Dz.U. 2019 poz. 1899. 